\title{
Los juegos populares como recurso didáctico para la mejora de hábitos de vida saludables en la era digital \\ Popular games as a teaching resource for the improvement of healthy living habits in the digital time \\ *Jesús López Belmonte, **Santiago Pozo Sánchez, **Arturo Fuentes Cabrera, **Manuel Ricardo Vicente Bujez \\ *Universidad Internacional de Valencia (España), **Universidad de Granada (España)
}

\begin{abstract}
Resumen. Las peculiaridades que presenta la sociedad actual junto a la imparable revolución tecnológica están provocando altos índices de sedentarismo, patologías y adicciones a los recursos digitales que nos rodean. Para hacer frente a esta situación, desde las instituciones escolares, se aboga por el fomento de juegos populares como vía de concienciación, motivación y promoción de hábitos de vida activos y saludables en los discentes. Así, el objetivo de esta investigación es analizar la incidencia de la práctica de juegos populares entre el alumnado de Educación Primaria. Para ello, se ha tomado una muestra de 3728 alumnos pertenecientes a 18 centros educativos de naturaleza cooperativa en la Comunidad Autónoma de Andalucía y la Ciudad Autónoma de Ceuta. En cuanto a los aspectos metodológicos se ha seguido un diseño no experimental de corte transversal, descriptivo y correlacional bivariado, a través de una metodología cuantitativa, empleando como instrumento un cuestionario ad hoc. Los resultados revelan que los discentes no siguen una vida activa fuera del horario escolar y manifiestan altos índices de adicción tecnológica. Además, se concluye que los juegos populares han contribuido a la mejora de la motivación, la satisfacción, la participación y el clima de aula en los distintos cursos analizados aunque no han conseguido dinamizar la colaboración de las familias. En relación con el género, se produce cierto dominio de las chicas en cuanto a la motivación, la participación, la cooperación y la adicción tecnológica frente a la satisfacción producida en los chicos.
\end{abstract}

Palabras clave: Juegos populares, herramienta pedagógica, vida saludable, hábitos activos, sedentarismo, adicción tecnológica.

\begin{abstract}
The peculiarities of today's society along with the unstoppable technological revolution are causing high rates of sedentary lifestyle, pathologies and addictions to the digital resources that surround us. To deal with this situation, from the school institutions, the promotion of popular games is advocated as a way of raising awareness, motivation and promotion of active and healthy life habits in the students. Thus, the objective of this research is to analyze the incidence of the practice of popular games among the students of Primary Education. For this, a sample of 3728 students belonging to 18 educational centers of a cooperative nature in the Autonomous Community of Andalucia and the Autonomous City of Ceuta was taken. Regarding the methodological aspects, a non-experimental cross-sectional, descriptive and bivariate correlation design was followed through a quantitative methodology, using as an instrument an ad hoc questionnaire. The results reveal that students do not follow an active life outside school hours and manifest high rates of technological addiction. In addition, it is concluded that popular games have contributed to the improvement of motivation, satisfaction, participation and classroom climate in the different courses analyzed although they have not been able to stimulate the collaboration of families. In relation to gender, there is a certain dominance of girls in terms of motivation, participation, cooperation and technological addiction compared to the satisfaction produced in boys.
\end{abstract}

Keywords: Popular games, pedagogic tool, healthy life, active habits, sedentary lifestyle, technological addiction.

\section{Introducción}

La enorme influencia que las tecnologías de la información y la comunicación (TIC) ejercen en el mundo actual ha provocado que estas se encuentren totalmente integradas en el seno de nuestras vidas. De esta forma, se ha originado un paradigma social en el que la tecnología se erige como facilitadora de las acciones llevadas a cabo en nuestra vida diaria. Sin embargo, es necesario destacar que las TIC han incidido — también — de forma negativa en la salud de sus usuarios, desembocando en problemas relacionados con un alto grado de sedentarismo (De Diego, Fernández \& Badanta, 2017) y con elevados niveles de adicción (Echeburúa \& De Corral, 2010).

Los problemas de salud detectados en la sociedad actual por los investigadores también se han manifestado en la población escolar (Avalos, Reynoso, Colunga, Oropeza \& González, 2017). Es por ello, que en el presente estudio se aborda como problema de investigación la existencia de una población estudiantil que presenta un alto riesgo de adop-

Fecha recepción: 04-10-18. Fecha de aceptación: 12-03-19

Jesús López Belmonte

jesus.lopezb@campusviu.es ción de hábitos de salud inadecuados y una utilización desmesurada de las TIC. En este sentido, un estilo de vida activo y saludable resulta fundamental para mantener la salud en estado óptimo, desembocando en la práctica de conductas beneficiosas para nuestro organismo (Asencio, Daza, Jiménez, Nájera \& Suárez, 2016). La escuela se erige, por tanto, como el escenario idóneo para promover la práctica diaria de actividad física (Sánchez, 2014) y fomentar la adquisición de una dieta completa y equilibrada en los discentes (Martín, Moreno \& Bes, 2015).

La obesidad es una de las enfermedades más frecuentes del siglo XXI. Esto es debido al enorme protagonismo que han tomado las tecnologías en la sociedad (González, Gómez \& Navarro, 2016) especialmente en los países desarrollados (San Mauro et al, 2015). Varios científicos, del ámbito de las Ciencias de la Salud, han constatado que un nivel alto de masa corporal (IMC) constituye un factor de riesgo determinante para la proliferación de trastornos cardiovasculares (Macaluso et al., 2002), enfermedades pulmonares, hepáticas y renales (De Onis, Blössner \& Borghi, 2010), enfermedades musculares y esqueléticas (Chan \& Chen, 2009), empeoramiento de la calidad de vida (Ul-Haq, Mackay, Fenwick \& Pel, 2013) y problemas en la socialización (Lobstein, Baur \& Uauy, 2004). En este mimo sentido, tal y como refieren Fonseca, Maldonado, Pardo \& Soto (2007), la Organización 
Mundial de la Salud ha llamado la atención sobre la obligación urgente de controlar las enfermedades de tipo cardiovascular mediante la concienciación de la sociedad para que incorporen a su vida diaria hábitos saludables. Macías, Gordillo \& Camacho (2012) entienden que deben ser las instituciones del ámbito de la educación las encargadas de crear políticas integrales que involucren a toda la comunidad educativa para que la consecución de hábitos beneficiosos para la salud se produzca de forma óptima y efectiva. Es por ello que la educación para la salud está adquiriendo cada vez mayor importancia en el sistema educativo actual con el fin de disminuir las acuciadas cifras de población infantil obesa (Vera, Sánchez \& Sánchez, 2018).

Otro factor determinante - ya mencionado- que contribuye de forma notable a los hábitos poco saludables de la población actual es la profusa incidencia del mundo tecnológico en la cotidianeidad de la sociedad. El nacimiento del smartphone ha supuesto un gran número de ventajas en nuestra vida diaria, pero también acarrea un importante número de riesgos (Pedrero, Ruiz, Rojo, Llanero, Morales \& Puerta, 2018) traducidos en grandes adicciones a infinidad de herramientas digitales como Internet (Young, 2017); teléfonos móviles (Pedrero, Rodríguez \& Ruiz, 2012), aplicaciones de mensajería instantánea (Dlodlo, 2015; Sultan, 2014), redes sociales (Schou, 2015), y juegos online (Griffiths, 2015). Además, se han encontrado correlaciones significativas entre la adicción a algunas de estas herramientas online y el desempeño negativo en labores cotidianas, baja autoestima y escaso rendimiento escolar (Grover et al., 2016).

A nivel legislativo, la Ley Orgánica 8/2013, de 9 de diciembre, para la mejora de la calidad educativa — normativa vigente en España en materia de educación - ha provocado un cambio paradigmático en el seno de la Educación Primaria. El corpus legislativo en materia de educación debe nutrirse de los avances científicos para la lucha contra el sedentarismo infantil y la promoción de una vida saludable (Méndez, Fernández-Río, Méndez \& Prieta, 2015). En este sentido, el currículo de Educación Física debe garantizar el desarrollo motriz de los escolares (Rosa, García \& Carillo, 2018) para que tengan la oportunidad de participar con un alto nivel de desenvolvimiento en actividades deportivas (Graber \& Locke, 2007) y así fomentar una vida activa lejos del sedentarismo.

Profundizando en la normativa vigente, el Real Decreto 126/2014, de 28 de febrero, por el que se establece el currículo básico de la Educación Primaria, establece en su artículo séptimo que uno de los objetivos de la etapa consistirá en «utilizar la educación física y el deporte como medios para favorecer el desarrollo personal y social» (p.41). Así mismo, se hace alusión especial a la importancia de los hábitos saludables dentro de la asignatura de Educación Física, ya que considera que dicha materia debe contribuir al trabajo competencial relacionado con el área de la salud, fomentando la práctica asidua de actividad física y promoviendo una actitud crítica hacia prácticas no saludables por parte de los discentes. Muñoz (2014) constata que los criterios de evaluación se conforman como auténticos objetivos de etapa. Así, uno de los criterios de evaluación establecidos en la normativa vigente estipula que el alumno debe ser capaz de solventar los retos inherentes al juego y a la práctica de actividad física, «aplicando principios y reglas para resolver las situaciones motrices, actuando de forma individual, coordinada y cooperativa y desempeñando las diferentes funciones implícitas en juegos y actividades» (p.41). Como puede observarse, la normativa contempla la importancia del juego como elemento de peso para el desarrollo discente. En un mayor nivel de concreción curricular, dentro del citado Real Decreto 126/2014, los estándares de aprendizaje vuelven a poner de manifiesto la importancia del juego en el currículo, al entender que el alumno debe ser capaz de reconocer «la riqueza cultural, la historia y el origen de los juegos y el deporte» (p.47), exponer «las diferencias, características y/o relaciones entre juegos populares, deportes colectivos, deportes individuales y actividades en la naturaleza» y realizar «actividades físicas y juegos en el medio natural o en entornos no habituales» (p.47).

Por lo que respecta a los juegos populares y tradicionales, resulta necesario un acercamiento a su concepto para comprender su alcance de forma integral. Los juegos son mecanismos básicos del proceso de socialización fundamentados en las características de la cultura en la que se inscriben (Delgado, Aguilera, Zamora, Oviedo \& Moreno, 2017; Rodríguez, Oliveira \& Navarro, 2019). Permiten fomentar relaciones entre los individuos, proyectadas en base a la dimensión cognitiva y socio-afectiva (Lavega, Lagardera, Molina, Planas, Costes, \& Sáez, 2006). Además, se han originado en el seno de la sociedad y se han transmitido de unas generaciones a otras (Mendoza, Tarpuk \& Lara, 2017; Torrebadella-Flix \& Domínguez, 2018) contribuyendo al proceso de socialización y cooperativismo de los individuos desde su infancia (Boyette, 2016) y pasando a formar parte de los rasgos identitarios de la comunidad (Burn, 2016). Así las cosas, las instituciones educativas deben luchar por la recuperación de los juegos populares propios de cada región y promover su práctica en base a un programa óptimo de actividad física (Ríos, Navarro, Arufe \& Pérez, 2018). En definitiva, deben desempeñar un rol fundamental en el currículo de Educación Primaria (Rodríguez, Pazos \& Palacios, 2016). En este sentido, Burn y Richards (2014) han constatado que los niños de Educación Primaria que practican juegos populares de forma asidua experimentan un importante desarrollo de la motivación, la autonomía y las relaciones interpersonales, además de contribuir de forma positiva a la satisfacción personal por la mejora de su estado físico. Dichos juegos pueden facilitar la resolución de conflictos de convivencia y eliminar los estereotipos de género de la mentalidad de los niños (Varela, 2015), favoreciendo el desarrollo de la capacidad empática, la resiliencia, las habilidades de socialización y el control y mejora de las emociones (Becerro \& Torrebadella, 2013; Zamorano, Gil, Prieto \& Zamorano, 2018). En este mismo sentido, varias investigaciones, entre las que destacamos Crespillo (2010); Baena y Ruiz (2016) han comprobado que la implantación de juegos populares en el ámbito escolar reduce las pérdidas de atención de los discentes y permite desarrollar su memoria al actuar como una herramienta motivadora que fomenta la participación del alumnado.

Por otra parte, aunque las investigaciones más recientes no han detectado diferencias reseñables en la correlación «vida saludable» y «tipo de centro» (Arufe, Chacón, Zurita, 
Lara \& Castro, 2017; Castro, Ríos-Reina, Ubeda \& Callejón, 2016; Morales, Martínez \& Díaz, 2016), es importante destacar que el presente estudio se ha llevado a cabo — concretamente- en un centro de naturaleza concertada que actúa en la sociedad como una cooperativa de enseñanza. Siguiendo a Martín, Fernández, Bel \& Lejarriaga (2013), el papel desempeñado por esta tipología de centros está ganando protagonismo durante los últimos años a pesar de su reciente incorporación, ocupando actualmente el tercer puesto en el ranking de modelos de escuela, por detrás de los centros públicos y privados. Fuentes, Lorenzo \& Corchón (2002) definen las cooperativas de enseñanza como instituciones educativas que persiguen la mejora del panorama educativo estatal a partir de modelos organizativos basados en la oferta de calidad, eficacia y vanguardia, aprovechando las potencialidades de su capital humano. Para conseguir una continuidad dentro de la panorámica de la educación, procuran sustentarse en un plan de formación continua del profesorado que compone su claustro docente (Fuentes, 2004; López, 2017; López \& Fuentes, 2018) encabezado por la figura del líder, un individuo afable, extrovertido, innovador y predispuesto a colaborar con cualquier trabajador para ayudarlo a cumplir sus objetivos (López, Fuentes \& Moreno, 2018).

Por todo lo expuesto, el objeto de estudio de la presente investigación se centra en implementar una unidad didáctica de Educación Primaria basada en juegos populares, para así promover una vida activa y sana que contribuya a la reducción del abuso tecnológico fuera del horario escolar y al fomento de hábitos de vida saludables.

En base a dicha intención, la investigación se ha centrado en analizar la incidencia de la puesta en práctica de juegos populares y tradicionales en discentes de Educación Primaria.

A partir de este objetivo principal se han formulado los siguientes objetivos específicos:

-Conocer el perfil de discentes de los centros educativos estudiados.

-Averiguar la motivación y satisfacción alcanzada por el alumnado durante el desarrollo de las sesiones.

-Conocer la participación de los discentes en los juegos realizados y su incidencia en el aprendizaje cooperativo.

-Constatar la contribución del juego popular a la consecución de los objetivos curriculares.

-Descubrir la incidencia del juego popular en el clima generado en los espacios de aprendizaje.

-Conocer la influencia del liderazgo discente dentro de su grupo de iguales.

-Comprobar el nivel de colaboración alcanzado por las familias en la implementación de juegos populares en la realidad de los alumnos.

-Determinar la influencia del género de los discentes con respecto a las distintas variables analizadas.

-Averiguar la asociación del curso al que pertenecen los discentes con su motivación, participación y cooperación en las tareas propuestas.

\section{Material y métodos}

\section{Contextualización}

Este estudio se ha llevado a cabo en 18 centros educati- vos de carácter concertado que siguen un modelo empresarial de cooperativa de enseñanza, localizados en la Comunidad Autónoma de Andalucía y la Ciudad Autónoma de Ceuta (España).

\section{Diseño}

Para alcanzar los objetivos fijados en esta investigación se ha optado por la elección de un diseño no experimental de corte transversal, descriptivo y correlacional bivariado, por medio de una metodología cuantitativa (McMillan \& Schumacher, 2005), con la finalidad de describir la realidad encontrada en el panorama educativo y cruzar diversas variables para obtener mayor consistencia en los resultados.

\section{Participantes}

Este estudio se ha centrado en la etapa de Educación Primaria (EP), de manera que la muestra utilizada en esta investigación se compone del total de alumnos correspondientes a los cursos $3^{\circ}, 4^{\circ}, 5^{\circ}$ y $6^{\circ}$ de la etapa de EP ( $\left.n=3728\right)$.

En la tabla 1 se detallan las características de los sujetos participantes referidas al género y edad de los estudiantes, mostrando estadísticos como número de alumnos (n), porcentajes (\%), población total $(\mathrm{N})$, media $\left(\mathrm{M}_{\mathrm{e}}\right)$ y desviación típica (DT) de los valores obtenidos.

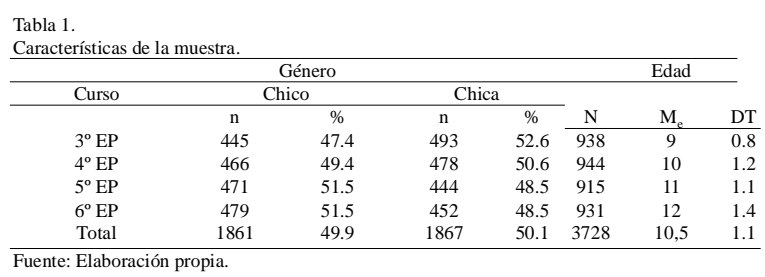

Los participantes de este estudio fueron escogidos siguiendo un muestreo dirigido o por conveniencia, debido a la facilidad de acceso a dicha muestra (Hernández, Fernández \& Baptista, 2014).

\section{Instrumento de recogida de datos}

Para llevar a cabo el proceso de recogida de datos ha sido necesario la utilización de un cuestionario ad hoc, confeccionado por los investigadores de este estudio con la finalidad de adaptar el instrumento a las necesidades y requerimientos de la investigación (Alaminos \& Castejón, 2006).

El cuestionario abarca un total de 26 ítems, estructurados en las siguientes dimensiones: a) Socio-demográfica (cinco ítems); b) Experiencia lúdica (ocho ítems); c) Hábitos saludables (doce ítems); d) Uso de la tecnología (seis ítems).

El formato de respuesta que sigue el instrumento es variado, ateniendo a las peculiaridades de la variable formulada, por lo que se encuentran respuestas tipo Likert con una escala de cuatro posibilidades (1-Nada, 2-Poco, 3-Bastante y 4-Totalmente), respuestas cerradas y otras de selección múltiple.

Para conocer la validez del instrumento se efectuó una validación de contenido por medio de un juicio de expertos (Escobar \& Cuervo, 2008), integrado por cinco Doctores especialistas pertenecientes al Departamento de Educación Física y deportiva de distintas universidades (Granada, Sevilla, Córdoba, Zaragoza y Madrid). El veredicto de los jueces fue positivo en ambos instrumentos y se tuvieron en 
cuenta las orientaciones y recomendaciones para mejorar la eficacia del mismo; no teniendo que realizar grandes modificaciones con respecto a la versión inicial de tal instrumento.

Posteriormente, se comprobó la fiabilidad del cuestionario por medio del alfa de Cronbach que ofreció unos resultados bastante elevados, alcanzando un á=.89 en la totalidad del instrumento que desglosado por las dimensiones que este contiene queda de la siguiente manera: Socio-demográfica: á=.893; Experiencia lúdica: á=.974; Hábitos saludables: á=.882; Uso de la tecnología: á=.811. Estos valores reflejan una alta consistencia interna en el instrumento a la vez que una alta fiabilidad al encontrarse el valor á entre .8-1 (Bisquerra, 2004).

Antes del comienzo del trabajo de campo se realizó una prueba piloto (Corral, 2009) en un centro educativo de características similares a los centros participantes, con la intención de optimizar el instrumento de recogida de datos.

\section{Variables}

Las variables que se han tomado para efectuar este estudio son: GEN (género de los discentes), CURS (curso de los discentes), MOTIV (motivación alcanzada por los alumnos en la realización de los juegos), SATIS (satisfacción de los estudiantes en el desarrollo de las sesiones), D-EXTRA (deporte 0 actividad física extraescolar realizada por el discente), ADITIC (adicción a la tecnología), PARTI-T (participación teórica en los ejercicios propuestos por los docentes), PARTI-P (participación práctica en los juegos realizados), ACOOP (aprendizaje cooperativo logrado durante la realización de las tareas), OBJET (grado de alcance de los objetivos curriculares, LÍDER (liderazgo discente en los juegos desarrollados), CLIM (clima generado en el espacio escolar) y COL (nivel de colaboración de las familias en las tareas propuestas).

\section{Procedimiento}

Esta investigación se inició en el curso académico 20172018 con la puesta en contacto de los investigadores con los equipos directivos y docentes de los Departamentos de Educación Física y Deportiva de las distintas cooperativas de enseñanza. Tras la explicación de los objetivos y pretensiones del estudio, se obtuvieron las autorizaciones de los centros educativos participantes para abordar tal pesquisa. En una segunda toma de contacto, se detalló y explicó a los docentes de Educación Física la unidad didáctica a desarrollar, así como su planificación y materiales necesarios.

La unidad didáctica de juegos populares denominada «Buscando en el baúl de los recuerdos» se llevó a cabo en los cursos de $3^{\circ}$ a $6^{\circ}$ de primaria, adaptando el nivel de los objetivos a las características del curso-grupo, con una temporalización para trabajar los contenidos de doce sesiones, en las que los discentes tomaron un rol activo, proponiendo juegos populares a los que jugaban sus familiares, fomentando de tal forma la participación y colaboración de las familias.

Una vez finalizada la unidad didáctica se comenzó el proceso de recogida de datos con la aplicación de los cuestionarios, con total anonimato de las respuestas (García, Alfaro, Hernández \& Molina, 2006) y bajo la colaboración de los docentes para que dicho proceso fuera eficaz, con la inten- ción de no alterar el normal transcurso del resto de sesiones.

Obtenida la información se procedió a su análisis para extraer los resultados más significativos que muestren la realidad actual y originen conclusiones relevantes para la comunidad científica.

\section{Análisis de datos}

Para el análisis estadístico descriptivo se ha obtenido el número de casos presentes en cada categoría y el porcentaje correspondiente. Para las variables cuantitativas se ha empleado la media ( $\mathrm{M}_{\mathrm{e}}$ ), la desviación típica (DT), el coeficiente de asimetría de Pearson ( $\mathrm{CA}_{\mathrm{p}}$ ) y el coeficiente de apuntamiento de Fisher $\left(\mathrm{CA}_{\mathrm{F}}\right)$. Además, la comparación de variables se ha llevado a cabo mediante la prueba Chi-cuadrado $\left(\dot{\bullet}^{\circ}\right)$ y V de Cramer ( $\mathrm{V}_{\text {Cramer }}$ )(Landero \& González, 2006). El análisis de los datos recogidos se efectuó con el software Statistical Package for the Social Sciences (SPSS) v.22, tomando un $p<.05$ como diferencias estadísticamente significativas entre las variables cruzadas.

\section{Resultados}

A continuación se presentan los resultados obtenidos tras el análisis estadístico realizado. En la tabla 2 se muestran los valores alcanzados en cada una de las variables, tanto de la escala Likert como los parámetros resultantes de los estadísticos efectuados.

En base a estas cifras, se hallan altos niveles en las variables motivación (MOTIV), satisfacción (SATIS), participación práctica (PARTI-P), clima de trabajo (CLIM) y adicción tecnológica (ADITIC). En cambio, las variables participación teórica (PARTI-T), aprendizaje cooperativo (ACOOP) y alcance de objetivos (OBJET) han recibido opiniones centrales, seguidas de las variables actividad física o deporte extraescolar (D-EXTRA), liderazgo (LÍDER) y colaboración familiar (COL) cuyos niveles han sido bajos.

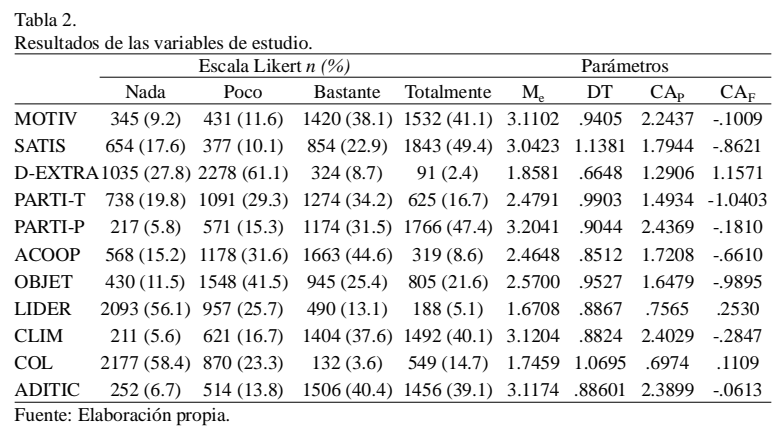

Con respecto a la asociación entre las variables analizadas y el género de los discentes (tabla 3), los resultados ofrecen relaciones estadísticamente significativas entre la variable género y la motivación (MOTIV: $p$-.0002), actividad física o deporte extraescolar (D-EXRA: p-.0001), alcance de objetivos (OBJET: p-.0521) y liderazgo discente (LÍDER: $p$ .0044). A pesar de dicho nivel de significancia, todas estas correlaciones ofrecen un escaso grado de dependencia con respecto al género de los discentes.

En cuanto a la asociación entre la motivación alcanzada por los discentes en el desarrollo de los contenidos y el curso en el que se encuentran estos, como se presenta en la 
Tabla 3.

\begin{tabular}{|c|c|c|c|c|c|}
\hline & \multicolumn{2}{|c|}{ Género $n(\%)$} & \multirow[b]{2}{*}{$? 2(\mathrm{gl})$} & \multirow[b]{2}{*}{$p$-valor } & \multirow[b]{2}{*}{$V_{\text {Crame }}$} \\
\hline & Chico & Chica & & & \\
\hline MOTIV & & & $16.68(1)$ & .0002 & \\
\hline $\mathrm{No}_{\text {MOTIV }}$ & 438 (11.75) & $338(9.07)$ & & & \\
\hline $\mathrm{Si}_{\text {MOTIV }}$ & $1423(38.17)$ & $1529(41.01)$ & & & \\
\hline $\begin{array}{l}\text { SATIS } \\
\text { SATI }\end{array}$ & & & $3.00(1)$ & .083 & .028 \\
\hline $\mathrm{No}_{\text {SATIS }}$ & $491(13,17)$ & $540(14,48)$ & & & \\
\hline Sí SATIS $_{\text {S }}$ & $1370(36.75)$ & $1327(35.60)$ & & & \\
\hline D-EXTRA & & & $26.94(1)$ & .0001 & .085 \\
\hline $\mathrm{No}_{\text {D-EXTRA }}$ & $1604(43.03)$ & $1709(45.84)$ & & & \\
\hline Sín & 257 (6.89) & $158(4.24)$ & & & \\
\hline PARTI-T & & & $.07(1)$ & .795 & .004 \\
\hline $\mathrm{No}_{\text {PARTLT }}$ & $917(24.6)$ & $912(24.46)$ & & & \\
\hline $\mathrm{Si}_{\text {PARTIT }}$ & $944(25.32)$ & $955(25.62)$ & & & \\
\hline $\begin{array}{l}\text { PARTI-P } \\
\text { PART-1 }\end{array}$ & & & $.14(1)$ & .710 & .006 \\
\hline $\mathrm{No}_{\text {PARTLP }}$ & 398 (10.68) & $390(10.46)$ & & & \\
\hline $\begin{array}{l}\text { Sí } 1_{\text {PARTILP }} \\
\text { ACOOP }\end{array}$ & $1463(39.24)$ & $1477(39.62)$ & $3.18(1)$ & .074 & .029 \\
\hline $\mathrm{No}_{\text {ACOOP }}$ & 894 (23.98) & $852(22.85)$ & & & \\
\hline $\mathrm{Si}_{\mathrm{ACOOP}}$ & $947(25.40)$ & $1015(27.23)$ & & & \\
\hline OBJET & & & $3.77(1)$ & .0521 & 0.32 \\
\hline $\mathrm{No}_{\text {OBJET }}$ & $1017(27.28)$ & $961(28.78)$ & & & \\
\hline Sí $_{\text {OBJET }}$ & $844(22.64)$ & $906(24.30)$ & & & \\
\hline LÍDER & & & $8.11(1)$ & .0044 & .047 \\
\hline $\mathrm{No}_{\text {LIDER }}$ & 1489 (39.94) & $1561(41.87)$ & & & \\
\hline $\mathrm{Si}_{\text {LIDER }}^{\prime}$ & $372(9.98)$ & $306(8.21)$ & & & \\
\hline $\mathrm{COL}$ & & & $2.89(1)$ & .0892 & .028 \\
\hline $\begin{array}{l}\mathrm{No}_{\mathrm{COL}} \\
\mathrm{SS}_{\mathrm{C}} 360(966)\end{array}$ & $1501(40.26)$ & $\begin{array}{r}1546(41.47) \\
321(8.61)\end{array}$ & & & \\
\hline $\begin{array}{l}\mathrm{Si}_{\mathrm{COL}}^{360}(9.66) \\
\text { ADITIC }\end{array}$ & & 321 (8.61) & $.74(1)$ & .389 & .014 \\
\hline $\mathrm{No}_{\text {ADTIIC }}$ & $393(10.54)$ & $373(10.02)$ & & & \\
\hline $\mathrm{Si}_{\text {ADETा }} 1468(39$ & .37) & $1494(40.07)$ & & & \\
\hline
\end{tabular}

tabla 4, no se ha obtenido una relación estadísticamente significativa puesto que no se ha obtenido un $p<.05$, pero a pesar de ello, se encuentran opiniones positivas en los diferentes cursos referidas a la motivación.

En consideración a la asociación entre la participación práctica del alumnado en las sesiones de trabajo y el curso en el que se encuentran, se afirma que se existe una relación estadísticamente significativa de baja dependencia, demostrado en los valores conseguidos en las pruebas $p$-valor y $\mathrm{V}_{\text {Cramer, }}$ como se visualiza en la tabla 5. Asimismo, se alcanzan opiniones positivas relacionadas con la participación práctica en los distintos cursos.

En la tabla 6, referida a la asociación entre la cooperación discente y el curso de estos, tampoco se determina una relación estadísticamente significativa debido al p-.59 obtenido. Por consiguiente, se muestran valores centrales de la escala Likert en las opiniones de los estudiantes con respecto a la cooperación en los juegos realizados.

\begin{tabular}{|c|c|c|c|c|c|c|}
\hline \multirow[b]{2}{*}{ Likert } & \multicolumn{3}{|c|}{ Curso $n(\%)$} & \multicolumn{3}{|c|}{ Parámetros } \\
\hline & $3^{\circ} \mathrm{EP}$ & $4^{\circ} \mathrm{EP}$ & $5^{\circ} \mathrm{EP}$ & $6^{\circ} \mathrm{EP}$ & ?2(gl) $p$-valor Cont. & $\mathrm{V}_{\mathrm{C} \text { Camer }}$ \\
\hline MOTIV & & & & & $2.04(9) \quad .9908 \quad .023$ & .04 \\
\hline Nada & $90(2.41)$ & $86(2.31)$ & $82(2.2)$ & $87(2.33)$ & & \\
\hline Росо & $108(2.9)$ & 117 (3.14) & $108(2.9)$ & $98(2.63)$ & & \\
\hline Bastante & $355(9.52)$ & 358 (9.6) & $352(9.44)$ & $355(9.52)$ & & \\
\hline Totalmente & 385 & $383(10.27)$ & $373(10.01)$ & ) $391(10.49$ & & \\
\hline \multicolumn{7}{|c|}{ Fuente: Elaboración propia. } \\
\hline \multicolumn{7}{|c|}{$\begin{array}{l}\text { Tabla } 5 . \\
\text { Asociación entre el n }\end{array}$} \\
\hline & \multicolumn{4}{|c|}{ Curso $n(\%)$} & Parámetros & \\
\hline Likert & $3^{\circ} \mathrm{EP}$ & $4^{\circ} \mathrm{EP}$ & $5^{\circ} \mathrm{EP}$ & $6^{\circ} \mathrm{EP}$ & ?2(gl) $\quad$-valor Cont. & $\mathrm{V}_{\text {Cramer }}$ \\
\hline 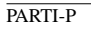 & & & & & $24.33(9) \quad .004 \quad .081$ & .140 \\
\hline Nada & $79(2.12)$ & $31(.83)$ & $52(1.39)$ & $55(1.48)$ & & \\
\hline Poco & $144(3.86)$ & $146(3.92)$ & $138(3.7)$ & $143(3.84)$ & & \\
\hline Bastante & $295(7.91)$ & 294 (7.89) & $291(7.81)$ & $294(7.89)$ & & \\
\hline Totalmente & $420(11.27)$ & $473(12.69)$ & 434 (11.64) & $439(11.78)$ & & \\
\hline \multicolumn{7}{|c|}{ Fuente: Elaboración propia. } \\
\hline \multicolumn{7}{|c|}{ Tabla 6.} \\
\hline \multicolumn{7}{|c|}{ Asociación entre el nivel de cooperación y el curso de los discentes. } \\
\hline & & Curso $n$ & & & Parámetros & \\
\hline Likert & $3^{\circ} \mathrm{EP}$ & $4^{\circ} \mathrm{EP}$ & $5^{\circ} \mathrm{EP}$ & $6^{\circ} \mathrm{EP}$ & ?2(gl) $\quad p$-valor Cont. & $\mathrm{V}_{\text {Cramer }}$ \\
\hline ACOOP & & & & & $7.46(9) \quad .59 \quad .045$ & .077 \\
\hline Nada & $149(4)$ & $142(3.81)$ & $135(3.62)$ & $142(3.81)$ & & \\
\hline Росо & $288(7.73)$ & $295(7.91)$ & $309(8.29)$ & $286(7.67)$ & & \\
\hline Bastante & 415 (11.13) & $422(11.32)$ & $410(11)$ & $416(11.16)$ & & \\
\hline Totalmente & $86(2.31)$ & $85(2.28)$ & $61(1.64)$ & $87(2.33)$ & & \\
\hline
\end{tabular}

\section{Discusión y conclusiones}

La presencia de las TIC en la sociedad de la información y el conocimiento ha provocado un aumento del sedentarismo, tal y como reflejan los resultados obtenidos en esta investigación en la que los discentes manifiestan que no suelen practicar actividad física o deporte fuera del horario escolar, hecho que repercute directamente en la salud y el bienestar de las personas (De Diego et al., 2017; Echeburúa \& De Corral, 2010; Pedrero et al., 2018; Young, 2017). Ante esta situación, los centros educativos deben fomentar prácticas saludables que contribuyan a un aumento de la actividad física de los discentes tanto dentro como fuera del horario escolar (Sánchez, 2014), mejorando de tal forma la salud (Asencio et al., 2016) y la implementación de un modo de vida más activo entre los jóvenes (Méndez et al., 2015).

El desarrollo de los dispositivos móviles ha sido uno de los grandes motivos que ha originado la rápida expansión de patologías cardiovasculares relacionadas directamente con la obesidad e inactividad de las personas (González et al., 2016). Siendo principalmente originadas por la adicción que sufren las personas a la tecnología, como expresan en sus estudios Pedrero et al., (2018) y Young (2017), hecho que también ocurre en este estudio en el que los discentes han manifestado altos índices de adicción tecnológica.

La utilización de recursos educativos como los juegos populares en el seno de la Educación Física se justifica en los estudios realizados por expertos como Ríos et al., (2018) que abogan por la recuperación y promoción de los juegos populares dentro del currículo que marca la legislación vigente en materia educativa. En la investigación realizada, la aplicación de sesiones con juegos populares como medio didáctico ha contribuido a la mejora de la motivación, la satisfacción, la participación práctica y el clima de aula, tal y como muestran investigaciones previas efectuadas por Baena \& Ruiz (2016), Burn \& Richards (2014). A pesar de ello, su empleo no ha ofrecido resultados favorecedores en el alcance de los objetivos marcados por los docentes y en la participación teórica que implicaban las sesiones de trabajo de manera complementaria.

Del mismo modo, el despliegue de un aprendizaje cooperativo a través de los juegos populares no ha alcanzado los mismos niveles de éxito que los obtenidos por Boyette (2016) y Delgado et al., (2017) en sus investigaciones, los cuales consideraron que esta naturaleza de recursos didácticos fomentaban la socialización y el cooperativismo.

Una de las finalidades propuestas en la investigación fue aumentar la colaboración e implicación de las familias como manifiestan Macías et al., (2012), sin embargo, los hallazgos resultantes han determinado que este sector de la comunidad educativa no ha participado en demasía durante el transcurso de las sesiones realizadas.

Del estudio realizado se concluye que ha habido predominancia del género femenino en la motivación, en la participación, en la cooperación y en la adicción a la tecnología, en cambio, el género masculino se ha declinado hacia la satisfacción interior que ha producido el trabajo mediante juegos populares. Asimismo, siguiendo en la misma línea, se han alcanzado índices negativos en la colaboración familiar, el liderazgo en las tareas y en la práctica deportiva extraescolar 
en el colectivo de las chicas, mientras que el alcance de los objetivos curriculares han sido inferiores en los chicos. Con respecto al curso en el que se encuentran los discentes, la motivación ha estado presente en todos ellos durante las distintas sesiones de trabajo, así como la participación activa de manera práctica. Sin embargo, el curso no ha resultado ser un factor determinante en el fomento de un aprendizaje cooperativo a través de los juegos populares como recurso didáctico.

En cuanto a las prospectivas de investigación se persigue que la utilización de juegos populares como elemento dinamizador de hábitos activos y saludables en los centros educativos redunde en la mejora y posterior aumento de la actividad física realizada por los estudiantes fuera del ámbito escolar, consiguiendo de tal manera que los jóvenes reduzcan las horas destinadas a la tecnología y tomen conciencia de la importancia de seguir una vida activa.

En cuanto a las limitaciones de este estudio se puede destacar la actitud de los discentes durante el proceso de recogida de información, pues algunos alumnos mostraron una escasa participación durante el proceso. En relación a la implicación del núcleo familiar, en este tipo de actividades académicas suele ser compleja, generalmente porque no aportan resultados visibles a corto plazo. El alumnado, en una línea similar, encuentra este tipo de estudios ajenos a su realidad y no obtienen un beneficio directo. Para poder solventar ambas cuestiones podrían organizarse jornadas provinciales apoyadas por las instituciones educativas y por la administración para hacer partícipes tanto al alumnado como a sus progenitores de los frutos obtenidos en investigaciones previas, además de poder evidenciar los beneficios que tiene la investigación educativa para la optimización de su propio proceso de enseñanza y aprendizaje y para la mejora de la salud.

Como futura línea de investigación se propone ampliar la muestra, tomando datos en centros educativos de naturaleza pública y privada, así como la ampliación a otras etapas educativas.

\section{Referencias}

Alaminos, A., \& Castejón, J. L. (2006). Elaboración, análisis e interpretación de encuestas, cuestionarios y escalas de opinión. Alicante: Marfil.

Arufe, V., Chacón, R., Zurita, F., Lara, A., \& Castro, D. (2017). Influencia del tipo de centro en la práctica deportiva y las actividades de tiempo libre de escolares. Revista Electrónica Educare, 21(1), 1-19.

Asencio, M. J., Daza, A., Jiménez, M., Nájera, Y., \& Suárez, M. (2016). Estilos de vida saludable en adolescentes relacionados con alimentación y actividad física: una revisión integrativa. Salud en movimiento, 8(1), 25-39.

Avalos, M. L., Reynoso, L., Colunga, C., Oropeza, R., \& González, Á. (2017). Relación del índice de masa corporal, actividades físicas y sedentarias en escolares. Revista Electrónica de Psicología Iztacala, 17(3), 978-996.

Baena, A., \& Ruiz, P. J. (2016). El juego motor como actividad física organizada en la enseñanza y la recreación. EmásF: revista digital de educación física, (38), 73-86.

Becerro, A., \& Torrebadella, X. (2013). El joc tradicional i popular a l'escola primària: una proposta de l'educació física cap a la cessió d'autonomia. REIRE Revista d'Innovació i Recerca en
Educació, 6(2), 100-118.

Bisquerra, R. (2004). Metodología de la investigación educativa. Madrid: La Muralla.

Boyette, A. H. (2016). Children's play and the integration of social and individual learning: A cultural niche construction perspective. Social learning and Innovation in contemporary hunter-gatherers, 13, 159-169.

Burn, A. (2016). Children's playground games in the new media age. Children's Games in the New Media Age, 15-44.

Burn, A., \& Richards, C. (2014). Children's games in the new media age: Childlore, media and the playground, Surrey, England, Ashgate Publishing Limited.

Castro, M., Ríos-Reina, R., Ubeda, C., \& Callejón, R. M. (2016). Evaluation of school menus: Comparing public, private and charter schools. Revista de Nutrição, 29(1), 97-108.

Chan, G., \& Chen, C. T. (2009). Musculoskeletal effects of obesity. Current opinion in pediatrics, 21(1), 65-70.

Corral, Y. (2009). Validez y confiabilidad de los instrumentos de investigación para la recolección de datos. Revista Ciencias de la Educación, 19(33), 228-247.

Crespillo, E. (2010). El juego como actividad de enseñanza aprendizaje. Gibralfaro Estudios Pedagógicos, (68), 14-19.

Delgado, D. V., Aguilera, M., Zamora, E. A., Oviedo, B. W., \& Moreno, C. (2017). Implementación del área de juegos populares en la finca experimental «La Represa» de la Universidad Técnica Estatal de Quevedo. Athlos: Revista internacional de ciencias sociales de la actividad física, el juego y el deporte, (12), 31-52.

De Diego, R., Fernández, E., \& Badanta, B. (2017). Uso de las TIC para fomentar estilos de vida saludables en niños/as y adolescentes: el caso del sobrepeso. Revista Española de Comunicación en Salud (RECS), 8(1), 79-91.

De Onis, M., Blössner, M., \& Borghi, E. (2010). Global prevalence and trends of overweight and obesity among preschool children. The American journal of clinical nutrition, 92(5), 1257-1264.

Dlodlo, N. (2015). Salient indicators of mobile instant messaging addiction with selected socio-demographic attributes among tertiary students in South Africa. South African Journal of Psychology, 45(2), 207-222.

Echeburúa, E., \& De Corral, P. (2010). Adicción a las nuevas tecnologías ya las redes sociales en jóvenes: un nuevo reto. Adicciones, 22(2), 91-95.

Escobar, J., \& Cuervo, A. (2008). Validez de contenido y juicio de expertos: una aproximación a su utilización. Avances en medición, 6(1), 27-36.

Fonseca, M., Maldonado, A., Pardo, L., \& Soto, M. F. (2007). Adolescencia, estilos de vida y promoción de hábitos saludables en el ámbito escolar. Umbral científico, (11), 44-57.

Fuentes, A. (2004). Las cooperativas de enseñanza:(un estudio de las cooperativas de trabajo asociado). Ceuta: Universidad Nacional de Educación a Distancia, Centro Asociado de Ceuta.

Fuentes, A. M., Lorenzo, M., \& Corchón, E. (2002). Las cooperativas de enseñanza como tercera vía dentro de nuestro sistema educativo: las cooperativas de trabajo asociado. Percepciones de sus directivos. Enseñanza \& Teaching, (20), 51-84.

García, F., Alfaro, A., Hernández, A., \& Molina, M. (2006). Diseño de Cuestionarios para la recogida de información: metodología y limitaciones. Revista Clínica de Medicina de Familia, 1(5), 232-236.

González, C. S., Gómez, N., \& Navarro, V. (2016). Programa de intervención educativa para el tratamiento de la obesidad infantil basado en videojuegos activos. Revista da FAEEBA-Educação e Contemporaneidade, 25(47), 155-172.

Graber, K. C. \& Locke, L. F. (2007). Are the national standards achievable? Conclusions and recommendations. Journal of Teaching in Physical Education, 26(4), 416-424. 
Griffiths, M. D. (2015). Online Games, Addiction and Overuse of. The International Encyclopedia of Digital Communication and Society, 1-6.

Grover, K., Pecor, K., Malkowski, M., Kang, L., Machado, S., Lulla, R., ... \& Ming, X. (2016). Effects of instant messaging on school performance in adolescents. Journal of child neurology, 31(7), 850-857.

Hernández, R., Fernández, C., \& Baptista, M. P. (2014). Metodología de la investigación. Madrid: McGraw Hill.

Landero, R., \& González, M. (2006). Estadística con SPSS y metodología de la investigación. México: Trillas

Lavega, P., Lagardera, F., Molina, F., Planas, A., Costes, A., \& Sáez, U. (2006). Los juegos y deportes tradicionales en Europa: entre la tradición y la modernidad. Apunts. Educación física y deportes, 3(85), 68-81.

Ley Orgánica 8/2013, de 9 de diciembre, para la mejora de la calidad educativa. $\mathrm{BOE} \mathrm{n}^{\circ}$ 295, de 10/12/2013.

Lobstein, T., Baur, L., \& Uauy, R. (2004). Obesity in children and young people: a crisis in public health. Obesity reviews, 5, 485.

López, J. (2017). El fenómeno del liderazgo en los centros de enseñanza de naturaleza cooperativa. En Alcántara, J.M., Bermúdez, M., Blanco, F.J., \& Heredia, J.M. (Eds.), Investigación e innovación en el ámbito universitario. Tendencias ante los retos actuales de la sociedad (pp. 37-46). Madrid: EOS.

López, J., \& Fuentes, A. (2018). El liderazgo aplicado a los modelos diferenciados en educación: El caso de las cooperativas de enseñanza. En Gairín, J., \& Mercader, C. (Eds.), Liderazgo y gestión del talento en las organizaciones (pp. 169-175). Madrid: Wolters Kluwer.

López, J., Fuentes, A., \& Moreno, A. J. (2018). El liderazgo efectivo en los centros concertados de naturaleza cooperativa: Percepciones de sus docentes. Actualidades Investigativas en Educación, 18(3), 1-21.

Macaluso, C. J., Bauer, U. E., Deeb, L. C., Malone, J. I., Chaudhari, M., Silverstein, J., et al. (2002). Type 2 diabetes mellitus among Florida children and adolescents. Public Health Reports. 117, 373-379.

Macías, A. I., Gordillo, L. G., \& Camacho, E. J. (2012). Hábitos alimentarios de niños en edad escolar y el papel de la educación para la salud. Revista chilena de nutrición, 39(3), 40-43.

Martín, N., Moreno, L., \& Bes, M. (2015). La importancia de la nutrición y los estilos de vida saludables en la infancia y adolescencia. In Anales del Sistema Sanitario de Navarra, 38 (3), 461-462.

Martín, S., Fernández, J., Bel, P., \& Lejarriaga, G. (2013). Necesidad de medidas para impulsar la creación de las empresas de participación desde los diferentes niveles de enseñanza. CIRIEC-España, Revista de Economía Pública, Social y Cooperativa, (78), 71-99.

McMillan, J. H. \& Schumacher, S. (2005). Investigación educativa. Madrid: Pearson.

Méndez, D., Fernández-Río, J., Méndez, A., \& Prieta, J. A. (2015). Análisis de los currículos autonómicos LOMCE de Educación Física en Educación Primaria. Retos: nuevas tendencias en educación física, deporte y recreación, (28), 15-20.

Mendoza, M. M., Tarpuk, E., \& Lara, L. (2017). Los juegos populares y su aporte didáctico en las clases de Educación física. EmásF: revista digital de educación física, (44), 79-93.

Morales, V., Martínez, A., \& Díaz, A. (2016). Participación en deporte escolar en la Región de Murcia: centros públicos versus concertados-privados. SPORT TK-Revista EuroAmericana de Ciencias del Deporte, 5(2), 47-52.

Muñoz, J. C. (2014). El currículo del área de Educación Física de Primaria en la LOMCE: Análisis del Real Decreto 126/
2014. EmásF: revista digital de educación física, (27), 24-39.

Pedrero, E. J., Rodríguez, M. T., \& Ruiz, J. M. (2012). Adicción o abuso del teléfono móvil. Revisión de la literatura. Mobile phone abuse or addiction. A review of the literature. Adicciones, 24(2), 139-152.

Pedrero, E. J., Ruiz, J. M., Rojo, G., Llanero, M., Pedrero, J., Morales, S., \& Puerta, C. (2018). Tecnologías de la Información y la Comunicación (TIC): abuso de Internet, videojuegos, teléfonos móviles, mensajería instantánea y redes sociales mediante el MULTICAGE-TIC. Adicciones, 30(1), 19-32.

Real Decreto 126/2014, de 28 de febrero, por el que se establece el currículo básico de la Educación Primaria. BOE nº 52 de 1/3/ 2014.

Ríos, Y., Navarro, R., Arufe, V., \& Pérez, J. A. (2018). Evaluación de un programa de actividad física mediante juegos populares en escolares de Educación Primaria. Retos: nuevas tendencias en educación física, deporte y recreación, 34, 108-113.

Rodríguez, J. E., Pazos, J. M., \& Palacios, J. (2016). La promoción de juegos y deportes populares y tradicionales: de los centros educativos al servicio municipal de deportes. El caso de Boiro. Revista Española de Educación Física y Deportes, (412), 79-92.

Rodríguez, J. E., Oliveira, B., \& Navarro, R. (2019). Análisis de las prácticas lúdicas y tradicionales en la en la zona norte del Camino Central Portugués y su relación con el ámbito educativo. Retos: nuevas tendencias en educación física, deporte y recreación, (35), 25-30.

Rosa, A., García, E., \& Carrillo, P. J. (2018). La educación física como programa de desarrollo físico y motor. EmásF: revista digital de educación física, (52), 105-124.

San Mauro, I., Megías, A., García, B., Bodega, P., Rodríguez, P., Grande, G., ... \& Garicano, E. (2015). Influencia de hábitos saludables en el estado ponderal de niños y adolescentes en edad escolar. Nutrición Hospitalaria, 31(5), 1996-2005.

Sánchez, J. (2014). Aplicación de Perseo desde el área de Educación Física. E-motion: Revista de Educación, Motricidad e Investigación 2, 88-150. Recuperado de http://www.uhu.es/publicaciones/ojs/index.php/e-motion/article/view/2380/2258

Schou, C. (2015). Online social network site addiction: A comprehensive review. Current Addiction Reports, 2(2), 175184.

Sultan, A. J. (2014). Addiction to mobile text messaging applications is nothing to «lol» about. The Social Science Journal, 51(1), 57-69.

Torrebadella-Flix, X., \& Domínguez, J.A. (2018). El deporte en la educación física escolar. La revisión histórica de una crítica inacabada. Retos: nuevas tendencias en educación física, deporte y recreación, (34), 403-411.

Ul-Haq, Z., Mackay, D. F., Fenwick, E., \& Pell, J. P. (2013). Metaanalysis of the association between body mass index and healthrelated quality of life among children and adolescents, assessed using the pediatric quality of life inventory index. The Journal of pediatrics, 162(2), 280-286.

Varela, A. G. (2015). Los juegos populares como herramienta para la convivencia en los recreos. Revista de estudios e investigación en psicología y educación, (2), 34-38.

Vera, F., Sánchez, E., \& Sánchez, J. (2018). Promoción de la actividad física saludable en el recreo escolar. Revista Internacional de Medicina y Ciencias de la Actividad Física y el Deporte, 18(72), 655-668.

Young, K. S. (2017). The evolution of Internet addiction. Addictive Behaviors, 64, 229-230.

Zamorano, M., Gil, P., Prieto, A., \& Zamorano, D. (2018). Emociones generadas por distintos tipos de juegos en clase de educación física. Revista Internacional de Medicina y Ciencias de la Actividad Física y el Deporte, 18(69), 1-26. 